\title{
Utilidad del factor estimulador de colonias de granulocitos (G-CSF) en episodios de neutropenia febril de alto riesgo en niños con cáncer
}

\author{
NATALIE RODRÍGUEZ Z., JUAN TORDECILLA C., \\ MYRIAM CAMPBELL B., PILAR JOANNON S., CARLOS RIZZARDINI L., \\ VERÓNICA SOTO A. y PATRICIA VERDUGO L.
}

\section{Usefulness of G-CSF in pediatric high risk cancer patients with fever and neutropenia}

Chemotherapy associated febrile neutropenia is an important cause of morbidity and mortality in pediatric patients with cancer. The use of granulocyte-colony stimulating factor (G-CSF) post chemotherapy decreases the risk of infectious complications but its efficacy during the febrile neutropenic episode remains controversial. Thirty five episodes of high-risk febrile neutropenia were randomized into two treatment arms, 18 received antibiotics and G-CSF (group A) and 17 received antibiotics only upon admission (group B). Both groups were comparable in terms of demographic and clinical characteristics. No significant differences between groups were found in duration of hospitalization (mean group A 7 vs group B 8 days), antibiotic treatment (mean 7 vs 8 days), fever ( 3 vs 2 days), nor of neutropenia (4 vs 3 days). One patient in group A died after RSV infection. Considering these results and a literature review, we propose that G-CSF should not be recommended in children during the course of their febrile neutropenic episode.

Key words: Pediatrics; Cancer; Febrile neutropenia episodes; Granulocyte colony stimulating factor.

Palabras claves: Pediatría; Cáncer; Episodios de neutropenia febril; Factor estimulador de colonias de granulocitos.

\section{Introducción}

Los esquemas de quimioterapia actualmente utilizados en cáncer infantil producen neutropenias intensas que se prolongan por espacio de 7 días en promedio. Las infecciones severas que se presentan en los períodos de neutropenia son una complicación frecuente en estos pacientes y una de las principales causas de mortalidad prevenible en este grupo ${ }^{1}$.

Se han realizado grandes esfuerzos para definir en forma precisa los factores clínicos y de laboratorio, que asociados al episodio de neutropenia febril (NF), representen riesgo de una infección sistémica invasora. En nuestro hospital se utiliza una clasificación de riesgo basada en dos estudios prospectivos realizados por el Comité de Infecciones del PINDA (Programa Infantil Nacional de Drogas Antineoplásicas) del Ministerio de Salud de Chile. De acuerdo a esta clasificación, aquellos pacientes que cursan con episodios de NF de alto riesgo, tienen una mayor probabilidad de presentar una infección sistémica de riesgo $\mathrm{vital}^{2,3}$.

El factor estimulador de colonias de granulocitos (colony stimulating factor- G-CSF), es una citoquina que regula la producción y maduración de los neutrófilos. Ha demostrado ser útil

\footnotetext{
Hospital de Niños Roberto del Río, Santiago, Chile:

Unidad de Hemato-Oncología (NRZ, JTC, MCB, PJS, CRL, VSA, PVL).

Facultad de Medicina. Universidad de Chile, Santiago, Chile:

Departamento de Pediatría. Campus Norte (NRZ, JTC, MCB, PVL).
}

Recibido: 16 noviembre 2004

Aceptado: 9 mayo 2005 
en la recuperación del valor de los neutrófilos que sucede al uso de quimioterapias intensas (uso profiláctico). Sin embargo, no ha sido aclarada su utilidad una vez instalado el episodio de $\mathrm{NF}^{4}$. La literatura médica señala que la administración de G-CSF durante el episodio febril acorta el período de neutropenia y el tiempo de hospitalización, aunque no en forma significativa en pacientes pediátricos ${ }^{5,6}$.

No se han publicado hasta ahora experiencias controladas en nuestro país acerca de la utilidad terapéutica de G-CSF en episodios de NF que cursan con infecciones severas. A pesar de esto, se estaba utilizando en algunos centros oncológicos en forma empírica frente a la potencial situación de gravedad de estos pacientes.

El presente estudio tiene como objetivo evaluar si el uso concomitante de G-CSF a tratamiento antimicrobiano en pacientes oncológicos pediátricos con episodios de NF de alto riesgo, reduce el período de neutropenia y hospitalización.

\section{Pacientes y Métodos}

En un estudio prospectivo randomizado, se incluyó a 28 pacientes que fueron internados en la Unidad de Oncología del Hospital de Niños Roberto del Río, por episodios de NF de alto riesgo, en un período de 20 meses, $\left(1^{\circ}\right.$ abril 2002 a 31 de diciembre 2003), totalizando 36 episodios.

\section{Definiciones}

Fiebre: temperatura axilar de $38^{\circ} \mathrm{C}$ en dos tomas separadas por 1 hora o una toma de $38,5^{\circ}$. Neutropenia severa: recuento absoluto de neutrófilos (RAN) menor o igual de $500 / \mathrm{mm}^{3}$.

Alto riesgo de infección invasora ${ }^{2,3}$ :

- Enfermedad de base: leucemia linfática aguda en recaída, leucemia mieloide aguda, neuroblastoma etapa 3 y 4 , linfoma de Burkitt o anaplásico de células gigantes y recaídas de tumores sólidos.

- Proteína $\mathrm{C}$ reactiva $\geq 90 \mathrm{mg} / \mathrm{Lt}$.

- Hipotensión arterial (de acuerdo a tablas de presión arterial según edad).

- $<7$ días desde el término del último ciclo de quimioterapia.

- Recuento de plaquetas $<50.000 / \mathrm{mm}^{3}$.

- Síndrome séptico.

La evaluación de los pacientes previa al inicio del tratamiento antimicrobiano incluyó historia clínica, examen físico, hemograma con recuento de plaquetas, PCR cuantitativa, cultivos de sangre obtenida por punción periférica y a través de catéter venoso central, orina completa y urocultivo, radiografía de tórax, otros cultivos (deposiciones, lesiones cutáneas, LCR, etc) y estudio por imágenes (ecografía abdominal) según fueran las manifestaciones clínicas.

En seguida, todos los pacientes iniciaron tratamiento antibacteriano endovenoso, según pautas de la Unidad de Oncología, con cloxacilina 50 $\mathrm{mg} / \mathrm{kg}$ cada $6 \mathrm{hrs}$, cefotaxima $40 \mathrm{mg} / \mathrm{kg}$ cada 6 hrs y amikacina $5 \mathrm{mg} / \mathrm{kg}$ cada $8 \mathrm{hrs}$. Se agregó metronidazol $5 \mathrm{mg} / \mathrm{kg}$ cada $8 \mathrm{hrs}$ si el paciente presentaba diarrea al ingreso o sospecha de enteropatía neutropénica ${ }^{7,8}$. Se indicó vancomicina $10 \mathrm{mg} / \mathrm{kg}$ cada 6 horas en forma empírica, como tratamiento de primera línea, sólo en pacientes portadores de catéter venoso central permanente que tenían el antecedente de haber presentado previamente una infección por cocácea grampositiva resistente a oxacilina.

Al momento de la hospitalización los pacientes fueron randomizados en dos grupos para recibir o no G-CSF en dosis de $5 \mu / \mathrm{kg} /$ día endovenoso asociado al esquema antimicrobiano. Se utilizó G-CSF elaborado por el Laboratorio Roche $\left(\right.$ Neupogen ${ }^{\circledR}$ ).

Ambos grupos de pacientes fueron evaluados diariamente en su estado general, curva febril, evolución del hemograma y de la PCR. Si la evolución clínica y de laboratorio no se consideraron favorables a las $72 \mathrm{hrs}$ de iniciado el tratamiento antimicrobiano (persistencia de fiebre y/o deterioro clínico asociado a aumento de PCR), se realizaron nuevos cultivos de sangre y otros exámenes en busca del foco infeccioso y el esquema terapéutico inicial fue cambiado a vancomicina $10 \mathrm{mg} / \mathrm{kg}$ cada $6 \mathrm{hrs}$ y ceftazidima $50 \mathrm{mg} / \mathrm{kg}$ cada 8 hrs manteniendo amikacina. Si a las 48 horas del cambio de esquema el paciente se mantenía febril o con mayor compromiso clínico, se suspendió ceftazidima y amikacina iniciando imipenem $15 \mathrm{mg} / \mathrm{kg}$ cada 6 horas. De persistir la fiebre hasta 7 días se adicionó anfotericina B deoxicolato 0,5 a $1 \mathrm{mg} / \mathrm{kg}$ cada $24 \mathrm{hrs}$. En caso de aislarse un microorganismo, este esquema terapéutico fue modificado de acuerdo a su patrón de susceptibilidad in vitro. Aquellos pacientes con bacteriemia, neumonía u otra infección bacteriana demostrada, completaron 10 días de tratamiento antiinfeccioso. En caso contrario, si el paciente evolucionaba afebril por más de 48-72 horas y sin agente etiológico identificado, se suspendió el tratamiento aunque persistiera neutropénico ${ }^{7,8}$.

Se consignó en cada episodio los días de hospitalización, de uso de antimicrobianos, de neutropenia severa, de fiebre y de PCR $>90 \mathrm{mg} / \mathrm{L}$. 
Fueron excluidos del estudio los pacientes que estaban recibiendo G-CSF profiláctico después de terminar su ciclo de quimioterapia.

Para calcular el tamaño de muestra nuestra hipótesis era que la evolución clínica en ambos grupos sería similar; considerando la variación de dos días como una diferencia aceptable en cada una de las variables analizadas, con un error tipo I de 0,05 y una potencia de 0,9 , se determinó un número de 30 pacientes por grupo. En el curso del estudio los diferentes protocolos de quimioterapia en uso fueron incorporando GCSF en forma profiláctica, de manera que el número de pacientes que cumplía con los criterios de inclusión se redujo considerablemente, por lo que se decidió cerrar nuestro estudio con la muestra presentada. Las variables analizadas fueron comparadas usando el test de $\chi^{2}$.

\section{Resultados}

En el período del estudio se incluyeron 36 episodios de NF catalogados de alto riesgo, en 28 pacientes. Uno de los episodios no se analizó en los resultados finales, por quiebre del protocolo, ya que se agregó G-CSF en la evolución del cuadro febril.

De los 35 episodios analizados, en 18 se administró G-CSF. Ambos grupos, con y sin G-CSF, presentaron características similares de edad, sexo, diagnóstico oncológico de base, presencia o no de catéter venoso permanente, uso de antibacterianos y antifúngicos profilácticos y necesidad de atención en Unidad de Tratamiento Intensivo (Tabla 1).

En 23 episodios el paciente presentaba un foco infeccioso identificable al ingreso, siendo principalmente respiratorio. Se aisló al menos un agente etiológico en 6 episodios que recibieron G-CSF y en 7 sin G-CSF, siendo más frecuentes, en los dos grupos, los bacilos gramnegativos (Escherichia coli y Klebsiella pneumoniae).

Fue necesario modificar el tratamiento antimicrobiano, utilizando esquemas de segunda o tercera línea, en 3 episodios tratados con G-CSF y en 8 de los episodios sin G-CSF, diferencia sin significación estadística.

La duración de la hospitalización en ambos grupos tuvo una mediana de 8 días (p: ns).

Tabla 1. Características clínicas comparativas de los grupos de estudio*

\begin{tabular}{lcc}
\hline Características & con G-CSF & sin G-CSF \\
\hline $\mathrm{N}^{\mathbf{o}}$ de episodios & 18 & 17 \\
Edad (años), mediana & $6(1-16)$ & $8(2-15)$ \\
Sexo (M/F) & $12 / 6$ & $8 / 9$ \\
Diagnóstico: & & \\
$\quad$ LLA recaída & 7 & 10 \\
LMA & 4 & 2 \\
$\quad$ Tumores sólidos & 7 & 5 \\
Catéter venoso central & $15(83 \%)$ & $12(70 \%)$ \\
Profilaxis antibacteriana y antifúngica & $15(83 \%)$ & $13(76 \%)$ \\
Foco infeccioso al ingreso & 13 & 10 \\
Hospitalización en UTI & 3 & 2 \\
\hline
\end{tabular}

LLA: Leucemia linfoblástica aguda; LMA: Leucemia mieloide aguda; UTI: Unidad de Tratamiento Intensivo; \# Uso de cotrimoxazol y nistatina oral. * p: $\mathrm{n} / \mathrm{s}$.

Tabla 2. Evolución clínica y de laboratorio con y sin uso de G-CSF*

\begin{tabular}{lccc}
\hline Duración de: (Días mediana) & Grupo total & Con G-CSF & Sin G-CSF \\
\hline Hospitalización & 8 & 8 & 7 \\
Tratamiento antimicrobiano & 7 & 8 & 7 \\
Neutropenia severa & 4 & 3 & 4 \\
Fiebre & 2 & 2 & 3 \\
PCR $>90 \mathrm{mg} / \mathrm{L}$ & 3 & 3 & 3 \\
\hline
\end{tabular}

*p: $\mathrm{n} / \mathrm{s}$. 
Tampoco se observó diferencias en la duración del tratamiento antimicrobiano, de la neutropenia, fiebre o de valor elevado de PCR (Tabla 2). Un paciente falleció a raíz de una infección severa por VRS.

\section{Discusión}

Se presenta la experiencia clínica en 18 episodios de NF de alto riesgo en que se asoció G-CSF al tratamiento antibacteriano. Nuestro estudio no logró demostrar que su uso produce reduce en forma significativa la duración de la hospitalización, del tratamiento antimicrobiano, de la fiebre ni de la neutropenia. En los episodios de NF tratados con G-CSF se modificó en menos ocasiones el esquema antibacteriano, no siendo necesario utilizar antimicrobianos de $2^{\mathrm{a}}$ ó $3^{\mathrm{a}}$ línea; sin embargo, esto no se tradujo en reducción del número de días de hospitalización. Esto puede deberse al número reducido de episodios analizados, pero permite sugerir que G-CSF no modifica la evolución clínica de estos episodios.

En 1996 la ASCO (American Society of Clinical Oncology) recomendó, en base a la evidencia disponible hasta ese momento, no utilizar en forma rutinaria factores estimuladores de colonias asociados al tratamiento anti-infeccioso para la mayoría de los pacientes que cursaban episodios de NF. Sin embargo, identificó factores asociados a un más probable deterioro clínico: presencia de neumonía, hipotensión arterial, disfunción multiorgánica (síndrome séptico) o infección fúngica severa; en ellos, consideró la ASCO razonable, el uso de G-CSF junto con la terapia anti-infecciosa, aunque especificó que no existía suficiente evidencia clínica para apoyar esta recomendación ${ }^{9}$.

A partir de 1997, diversos estudios clínicos realizados en pacientes pediátricos han mostrado algunos beneficios. El reporte con mayor número de casos, 186 pacientes con leucemia, linfoma y tumores sólidos, demostró una reducción de 2 días en el período de hospitalización y de 1 día en la duración del tratamiento antimicrobiano con la adición de G-CSF${ }^{5}$. Un segundo estudio realizado con factor estimulador de granulocitos y macrófagos: GM-CSF (granulocyte-macrophage colony stimulating factor), no logró demostrar disminución en la duración de la neutropenia pero se observó que, en el grupo de pacientes con GM-CSF, la hospitalización fue más breve ${ }^{6}$.

Sin embargo, a la luz de estos estudios pediátricos y otros estudios en adultos, la ASCO recomendó, en el año 2000, que G-CSF y GM-CSF no deberían ser utilizados en forma rutinaria asociados a tratamiento antimicrobiano en pacientes cursando episodios de NF.

Considerando el costo económico que representa el uso de estos factores estimuladores de colonias, y basados en la revisión de la literatura publicada hasta ahora, más los resultados de nuestra serie clínica, consideramos que no estaría recomendado el uso de G-CSF en el curso de episodios de NF de alto riesgo y que su gran utilidad sigue siendo en la profilaxis primaria, es decir, en su utilización después de la administración de quimioterapia mielosupresora con riesgo conocido de producir un episodio de NF. Sin embargo, en casos críticos puede considerarse su indicación ${ }^{4,9}$.

Agradecimientos: A las enfermeras universitarias de la Unidad de Oncología, a los médicos residentes de Pediatría y de Hemato-Oncología del Hospital Roberto del Río.

\section{Resumen}

La neutropenia febril (NF) secundaria a quimioterapia es causa importante de morbilidad y mortalidad en los pacientes oncológicos pediátricos. El uso de factor estimulante de colonias de granulocitos (GCSF) después de ciclos de quimioterapia intensa ha disminuido la frecuencia de complicaciones infecciosas asociadas, pero su utilización durante el episodio de NF es controvertida. Se analizaron 35 episodios de NF de alto riesgo. En forma randomizada 18 pacientes recibieron G-CSF asociado al tratamiento antimicrobiano habitual (grupo A) y 17 no lo recibieron (grupo B). Ambos grupos tenía parámetros biomédicos y clínicos similares. No se encontró diferencias significativas con respecto a la duración de la hospitalización (promedio grupo A 8 días vs grupo B 7 días) ni del tratamiento antimicrobiano (promedio 8 vs 7 días), de la fiebre (promedio 2 vs 3 días) y del período de neutropenia (promedio 3 vs 4 días). Considerando la revisión de la literatura y la experiencia local creemos que el uso de G-CSF no estaría recomendado en el manejo de pacientes oncológicos con episodios de NF.

\section{Bibliografía}

1.- Pizzo P A. Management of fever in patients with cancer and treatment-induced neutropenia. N Engl J Med 1993; 328: 1323-32.

2.- Santolaya M E, Álvarez A M, Becker A, Cofré J, Enríquez N, O’Ryan M, et al. Prospective, multicenter evaluation of risk factors associated with invasive bacterial infection in children with cancer, neutropenia and fever. J Clin Oncol 2001; 19: 3415- 21. 
3.- Santolaya M E, Álvarez A M, Avilés C L, Becker A, Cofré J, Enríquez N, et al. Prospective evaluation of a model of prediction of invasive bacterial infection risk among children with cancer, fever and neutropenia. Clin Infect Dis 2002; 35: 678-83.

4.- Clark O A, Lyman G, Castro A A, Clark L G, Djulbegovic B. Colony stimulating factors for chemotherapy induced febrile neutropenia. The Cochrane Database of Syst Rev 2003; CD 003939. Review.

5.- Mitchell P, Morland B, Stevens M C, Dick G, Easlea D, Meyer L C, Pinkerton C R. Granulocyte colonystimulating factor in established febrile neutropenia: a randomized study of pediatric patients. J Clin Oncol 1997; 15: 1163-70.

6.- Riikonen P, Saarinen U, Makipernaa A, Hovi L, Komulainen A, Pihkala J. Recombinant human granulocyte-macrophage colony-stimulating factor in the treatment of febrile neutropenia: a double blind placebo-controlled study in children. Pediatr Infect Dis J 1994; 13: 197-202.

7.- Tordecilla J, Campbell M, Joannon P, Rodríguez N. Alta precoz de niños con cáncer y neutropenia febril. Rev Chil Pediatr 1994; 65: 260-3.

8.- Tordecilla J, Campbell M, Joannon P, Rizzardini C, Soto V. Criterios de alta precoz en niños con cáncer y neutropenia febril. Rev Chil Pediatr 1998; 69: 247-51.

9.- Ozer H, Armitage J O, Bennett CL, Crawford J, Demetri G D, Pizzo P A, et al. 2000 Update of recommendations for the use of hematopoietic colonystimulating factors: evidence-based, clinical practice guidelines. American Society of Clinical Oncology Growth Factors Expert Panel. J Clin Oncol 2000; 18: 3558-85.

Correspondencia a:

Natalie Rodríguez Zamora. nrodriguez@med.uchile.cl 